\title{
Toward Systematic Approaches to Design and Implement Vehicles Semi-Active Control Systems
}

\author{
Hamidreza Bolandhemmat \\ PhD Candidate, Mechanical and Mechatronic \\ Engineering Department \\ University of Waterloo, Ontario, Canada \\ Email: hrboland@uwaterloo.ca
}

\author{
Farid Golnaraghi \\ Professor and Director, Mechatronic Systems Engineering, Simon Fraser University, \\ Surrey, BC, Canada, V3T 0A3 \\ Tel.: (778) 782-8054 Fax: (778) 782-7514 \\ Email: mfgolnar@sfu.ca
}

\begin{abstract}
In this paper a systematic while practical methodology has been presented for design of vehicle's semiactive suspension systems. The semi-active control strategies developed to improve vehicle ride comfort and stability generally have a switching nature. This makes the design of the controlled suspension systems difficult and highly dependent on an extensive trial and error process. The proposed methodology maps the discontinuous control system model to a continuous linear region where all the time/frequency design techniques established in the conventional control system theory can be applied. If the semiactive control system is designed to satisfy some ride/stability requirements, an inverse mapping offers control law. The effectiveness of the proposed design methodology in dealing with real industrial problems is demonstrated with experimental results.
\end{abstract}

\section{INTRODUCTION}

In the previous generation of vehicles, the suspension systems were designed and manufactured with fixed properties. Unfortunately, such systems do not provide an optimal ride or an acceptable level of stability under all circumstances.

For several decades, controllable suspension systems including active and semi-active systems have been developed to concurrently improve the ride comfort, road handling and stability of terrain vehicles. However, active suspension mechanisms were soon abandoned by vehicle manufacturers due to high cost, implementation complexities and also failure mode safety issues.

Semi-active suspension systems are supplied only by a low power electrical signal and exhibit high performance vibration isolation. The command signal may either vary the oil flow rate between the compression chamber and the reverse chamber of the semi-active damper (e.g. dampers with solenoid valves) or change the properties of the material inside the shock (e.g. Magneto-rheological (MR) dampers) to provide different level of resisting forces.

Various control methodologies have also been proposed to determine the desired damping force. The Skyhook control strategy introduced by Karnopp et al. [1] is undoubtedly the most widely used control policy for semi-active suspension systems. Following the Skyhook policy, the semi-active damper emulates a fictitious damper behavior mounted between the sprung mass and a stationary sky. It has been established that the Skyhook strategy can significantly attenuate the resonant peak of the sprung mass causing enhancement to the vehicle's ride comfort.

However, the Skyhook strategy does not address issues associated with wheel vibration. As a result, the technique can not decrease the resonant peak that corresponds to the unsprung mass; offering less improvement on the handling performance and stability of the vehicle. To overcome the disadvantage of the original Skyhook concept, Novak et al. [2] added another fictitious damper between the unsprung mass and the ground. This increases traction between the vehicle tire and the ground to enhance the handling characteristics of the vehicle.

Both the original Skyhook strategy and its modified version, the Skyhook-Groundhook control named as hybrid control, are also effective in terms of the simplicity of the control algorithm. Their other advantage is the fact that except some preliminary information regarding the vehicle's shocks characteristics, they do not require any a priori knowledge to explain the dynamics of the vehicle. That is, they are not model based.

The Skyhook policy can be either applied as a bang-bang controller or in a continuous manner by utilizing the sprung mass vertical velocity feedback (however, this version is also discontinuous). The on-off Skyhook controller is usually simpler and better suited for the industrial applications. The control law can be described as simple as

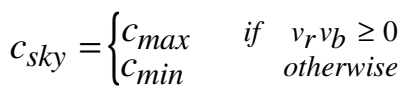


in which $v_{b}$ is the absolute velocity of the sprung mass and $v_{r}$ is the relative velocity of the sprung and unsprung mass across the suspension. Extensive theoretical and experimental study on the performance of different types of semi-active Skyhook (-Groundhook) controllers can be found in the literature [3-10].

Nevertheless, the controller upper and lower gains i.e. $c_{\max }$ and $c_{\text {min }}$ are usually determined by trial and error and there is no systematic method to adjust them. This would make the controllable suspension system development process time consuming and sometimes too hard. The current work aims to introduce a new methodology which allows for systematic design and implementation of the on-off Skyhook control strategy for semi-active suspensions.

The discontinuous nature of semi-active control strategies including the Skyhook policy is the main barrier to methodical design. The first step of the new methodology being presented employs fuzzy system theory to create a network with continuous valued outputs to emulate the discontinuous controller law. Once the original control strategy is converted to a continuous form, the different well-established frequency or time domain techniques can be employed to design and adjust suspension system controller parameters.

The remainder of the manuscript is organized in 5 sections. First in section II, structure of a general semi-active suspension system is presented and a proper analytical model is assigned to each single element of the closed loop control system. The new design methodology is introduced in Section III. Finally, real time implementation results and the corresponding analysis are given in the last section.

\section{Structure of THE Closed Loop Semi-ACtive Control SYSTEM}

Fig. 1 shows a typical configuration of the vehicle's semiactive suspension system. Vector $z$ contains outputs of sensors strategically distributed throughout the vehicle to capture vehicle's motions. The information sent by the sensors is processed by either an estimator e.g. an Extended Kalman Filter (EKF) or an ordinary low/high pass digital filter. The outputs of the filtering unit are the vehicle states required for the semi-active controller. Incorporating the vehicle states, the Skyhook control strategy determines the damping characteristic suitable for the current time-step. The control command is accordingly fulfilled by the vehicle semi-active shocks.

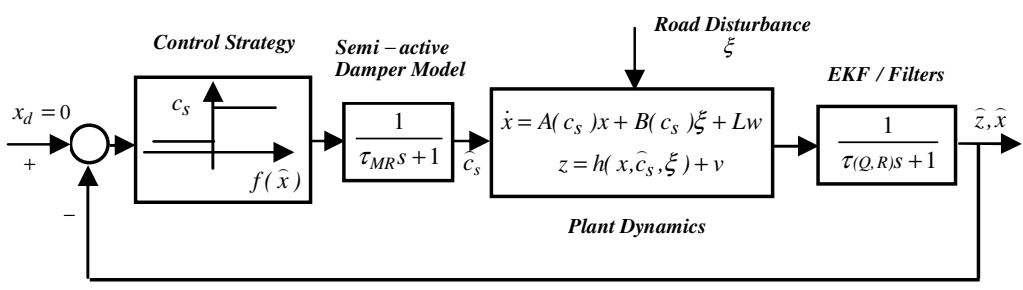

Fig. 1. Block diagram of the vehicles semi-active suspension control system.
The semi-active damper behaves like a low pass filter with a relatively high bandwidth. The bandwidth corresponding to the MR dampers can be experimentally achieved [11], [12] to be approximately $65(\mathrm{rad} / \mathrm{sec})$. The vehicle dynamics may be modeled as a linear time-varying (with respect to the semiactive damping coefficient $c_{S}$ ) system of the following form:

$$
\dot{x}=A\left(c_{s}\right) x+B\left(c_{s}\right) \xi+L w
$$

where $x(t)$ is the vehicle state vector and $\xi$ stands for the road input. To account for uncertainties existing in the analytical model, a white noise signal $w(t)$ with strength of $Q(t)$ may be also considered as an extra input to the model. In addition, $A, B$ and $L$ are the corresponding state, road input and the noise input matrices.

The measurement system model, (3), is typically given as a nonlinear combination of the vehicle states, the current damping coefficient and the road input.

$$
z=h\left(x, c_{s}, \xi\right)+v
$$

The sensor data is also assumed to be corrupted by a zero mean white noise $v$ having a covariance of $R$.

By employing a proper sensor configuration, most of the vehicle's required states can be observed through the EKF [13]. In this case, the EKF simply appears as a low pass filter whose bandwidth would be a function of $Q$ and $R$ matrices. Otherwise, the combination of a low pass filter and a differentiator or an integrator following by a high pass filter can be utilized to exploit the required states. In either circumstance, the filtering block can be easily substituted by a suitable transfer function.

The discontinuous block in Fig. 1 describing the Skyhook policy is the only module that can not be classified in the framework of conventional control system theory. The new approach aims to bring the discontinuous control strategy into the general framework of the classic control theory, so that all conventional control system theory tools can be employed in the design of a semi-active suspension controller. The next section pursues this goal.

\section{FUZZY NETWORK MODEL OF SKYHOOK}

It can be proven that a fuzzy logic network is capable of approximating any non-linear function on a compact set to an arbitrary accuracy [14]. Thus, a continuous fuzzy system can be developed to mimic the on-off Skyhook control strategy and maintain the controller efficiency. One method to create such a network is to encapsulate the Skyhook control logic into the inference engine of the fuzzy network. The fuzzy inference engine is the brain of the fuzzy system which induces a fuzzy output based on a predefined fuzzy rule base. In this case, the rule base built on the Skyhook strategy consists of the following 4 rules 
$\mathrm{R}^{1}:$ IF $v_{b}$ is "P" AND $v_{r}$ is "P" THEN $c_{s}$ is "B"
$\mathrm{R}^{2}:$ IF $v_{b}$ is "N" AND $v_{r}$ is "P" THEN $c_{s}$ is "S"
$\mathrm{R}^{3}:$ IF $v_{b}$ is "P" AND $v_{r}$ is "N" THEN $c_{s}$ is "S"
$\mathrm{R}^{4}:$ IF $v_{b}$ is "N" AND $v_{r}$ is "N" THEN $c_{s}$ is "B",

where $\mathrm{P}$ and $\mathrm{N}$ are the primary fuzzy sets defined in the $\mathbb{R}$ which is the universe of discourse of the input variables $v_{b}$ and $v_{r}$. P standing for "Positive" and $\mathrm{N}$ for "Negative" is selected as characteristic equations (4) and (5).

$$
\begin{aligned}
& \mu_{p}\left(v_{i}\right)=\frac{\kappa}{1+\exp \left[-\left(v_{i}-\gamma\right) / q\right]} \\
& \mu_{N}\left(v_{i}\right)=\frac{\kappa}{1+\exp \left[\left(v_{i}-\gamma\right) / q\right]}
\end{aligned}
$$

in which $i=b, r$. Inputs to the fuzzy system are normalized such that the same membership functions can be employed on both inputs. The normalized crisp input vector $v=\left[\begin{array}{ll}v_{b} & v_{r}\end{array}\right]^{T}$ is then fuzzified via a Singleton fuzzifier [14] as shown in Fig. 2.

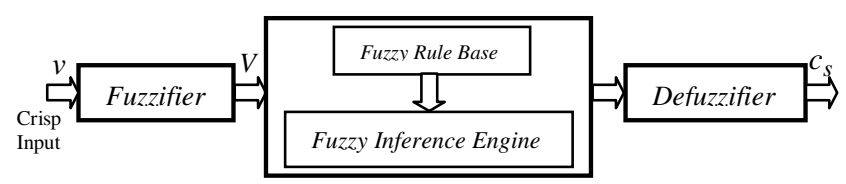

Fig. 2. Structure of the fuzzy logic system.

By employing the product operation rule of fuzzy implication [14] and the sup-product compositional rule [14], [15] the fuzzy inference engine concludes a single output per each IF-THEN rule of the fuzzy rule base from the fuzzy input vector $V$. Final output of the fuzzy inference engine is the union of each rule's outcome. The fuzzy output set $C_{s}$ is then converted into a crisp output by a Center Average Defuzzifier [14]. It can be shown that the input-output relation of the fuzzy controller can be written in the following closed form formula

$$
c_{S}=W^{T} \cdot \bar{C}
$$

where $W=\left[W^{\ell 1}\right]$ for $\ell=1, \ldots, 4$. Further, $W^{\ell 1}$ is defined by (7).

$$
W \ell=\frac{\prod \mu_{u_{j}^{\ell}}\left(v_{b}\right) \cdot \mu_{u_{k}^{\ell}}\left(v_{r}\right)}{\sum_{\ell \ell=1}^{4} \Pi \mu_{u_{j}^{\ell \ell}}\left(v_{b}\right) \cdot \mu_{u_{k}^{\ell \ell}}\left(v_{r}\right)}
$$

in which $j, k$ may be either $\mathrm{P}$ or $\mathrm{N}$ depending upon the rule number, $\ell$. Similarly, $\bar{C}=\left[\bar{c}_{S}^{\ell 1}\right]$ with $\bar{c}_{S}^{\ell 1}$ equals with either $c_{\max }$ or $c_{\min }$.

Having employed the fuzzy controller (6), the structure of the semi-active suspension control system is illustrated by Fig. 3 , where the on-off controller has been replaced by the continuous nonlinear map $N$ defined by (8).

$$
c_{s}=N\left(\hat{x}, c_{\max }, c_{\min }\right)
$$

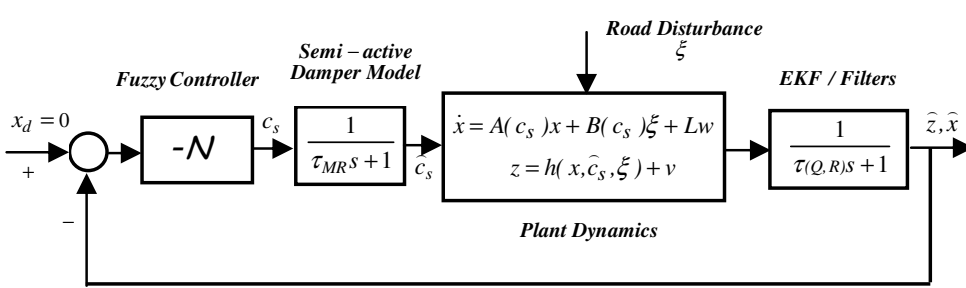

Fig. 3. The semi-active suspension control system with the Fuzzy Skyhook controller.

In the next step, the only nonlinear element of the control $\operatorname{system} N$ is expanded around a working point $x_{0}$ in which

$$
v_{b_{0}}=v_{r_{0}}=\gamma
$$

where $\gamma$ is the offset value used in (4) and (5). Considering the Taylor series expansion formula, the nonlinear function can be described as follows

$$
N \cong N\left(x_{0}\right)+\left.\frac{\partial N}{\partial x_{0}}\right|_{x_{0}} ^{T}\left(x-x_{0}\right) .
$$

Use of equations (4), (5) and (7) after some mathematical manipulation, it can be shown that

$$
\left.\frac{\partial N}{\partial x_{0}}\right|_{x_{0}}=0
$$

due to symmetry of the membership functions defined on the inputs i.e., (4) and (5). Similarly, $N\left(x_{0}\right)$ is obtained as

$$
N \cong N\left(x_{0}\right) \cong \frac{c_{\max }}{r(\kappa, q)}
$$

Since $c_{\min }$ must be selected much less than $c_{\max }$ (zero in theory), terms containing $c_{\min }$ have been ignored in (12). Further, $r$ is a constant which relates to the membership functions parameters $\kappa$ and $q$.

By concatenating the loop transfer functions and applying (12), the state space realization of the suspension control system is written as

$$
\dot{x}_{s}=A_{s} x_{s}+B_{s} u_{s}+L_{s} w_{s}
$$

where the state ,input and disturbance vectors are given in (14)

$$
x_{s}=\left[\begin{array}{c}
x \\
\widehat{x} \\
\hat{c}_{s}
\end{array}\right], u_{s}=\frac{c_{\max }}{r(\kappa, q)}, w_{s}=\left[\begin{array}{c}
\xi \\
w
\end{array}\right]
$$

and the corresponding matrices are as follows 


$$
\begin{gathered}
A_{s}=\left[\begin{array}{ccc}
A\left(\hat{c}_{s}\right) & 0 & 0 \\
\tau^{-1}(Q, R) & -\tau^{-1}(Q, R) & 0 \\
0 & 0 & -\tau_{M R}^{-1}
\end{array}\right], \quad B_{s}=\left[\begin{array}{c}
0 \\
0 \\
\tau_{M R}^{-1}
\end{array}\right] \\
L_{s}=\left[\begin{array}{cc}
B\left(\hat{c}_{s}\right) & L \\
0 & 0 \\
0 & 0
\end{array}\right] .
\end{gathered}
$$

Now, the original switching control system has been approximated by a linear state space model described by (13) to (15). Subsequently, all the tools well-known in the conventional control system theory from pole placement, LQR/LQG and $H_{2 / \infty}$ to various frequency domain techniques can be utilized to design the unknown parameter $c_{\max }$ such that the closed loop control system satisfies some desired requirements. In practice, the nonlinear controller (8) incorporating the designed $c_{\max }$ is implemented. Even though, $c_{\max }$ obtained by the proposed methodology needs to be finetuned during real time road tests to assure for the required ride comfort and stability.

\section{CASe Study and Real Time Results}

To demonstrate the proposed methodology, a Cadillac SRX 702005 equipped with semi-active MR dampers was selected as the experimental test bed. Some of the Cadillac SRX specifications are listed in table 1 [16].

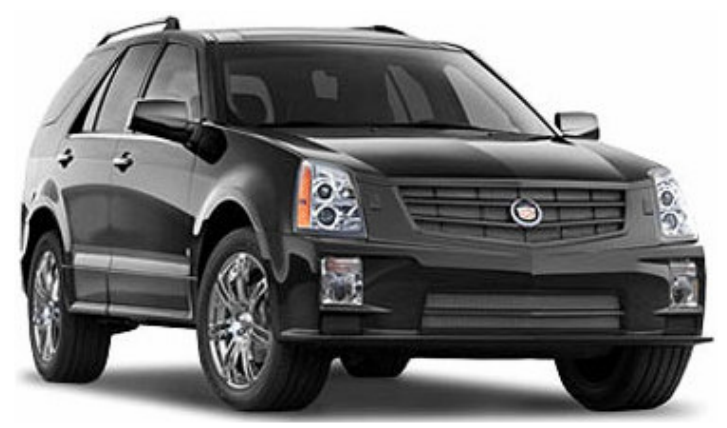

Fig. 4. The Cadillac SRX 70 test vehicle.

The MR dampers of the Cadillac SRX were tested in the University of Waterloo's MTS test facility to characterize the corresponding damping forces with respect to the applied control current and the relative velocity of the vehicle chassis to the wheels. It turned out that the MagneRide ${ }^{\mathrm{TM}}$ MR dampe is capable of providing damping coefficients ranging fron 200 N.sec/m (@0 Amp) to about 7000 N.sec/m (@5 Amp).

The test vehicle was also instrumented with a distributed sensor configuration consisting of 8 accelerometers, 1 IMU and 4 displacement sensors. The displacement sensors (see Fig.5) already installed by the vehicle manufacturer measure the relative displacement of the body and each wheel hop across the shock.
TABLE I

CADILLAC SRX 70 EXTERIOR DIMENSIONS

\begin{tabular}{|c|c|}
\hline Wheelbase $(\mathrm{mm})$ & 2957 \\
\hline Overall length $(\mathrm{mm})$ & 4950 \\
\hline Overall width $(\mathrm{mm})$ & 1844 \\
\hline Overall height $(\mathrm{mm})$ & 1722 \\
\hline Track $(\mathrm{mm})$ & $\begin{array}{c}\text { Front: } 2957 \\
\text { Rear: } 1580\end{array}$ \\
\hline Base Curb Weight $(\mathrm{kg})$ & 2013 \\
\hline Min. ground Clearance $(\mathrm{mm})$ & 208 \\
\hline Weight Distribution $(\%$ front/rear) & $52 / 48$ \\
\hline
\end{tabular}

As introduced by (1), the Skyhook control strategy requires information of the sprung mass motion as well as its relative motion with respect to the wheels. Therefore, two accelerometers were installed at the ends of each damper, one on the body end and the other close to each wheel hop totaling 8. Fig. 6 shows an accelerometer mounted near the wheel hop. The IMU was mounted in the vicinity of the vehicle's Center of Gravity (CG) and measures the 6 Degree of Freedom (DOF) body movement.

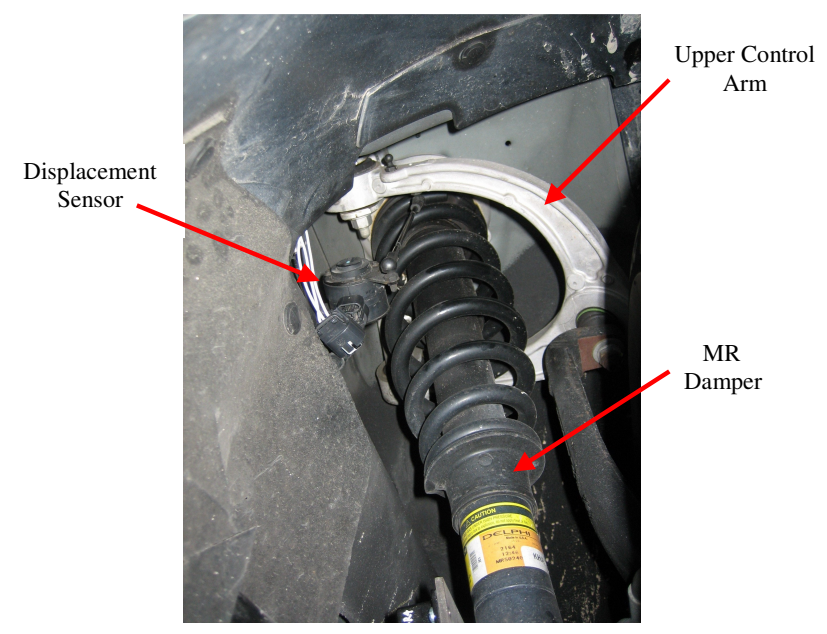

Fig. 5. The Cadillac SRX suspension compartment.

An EKF was developed to fuse sensors measurements and estimate the required states by the vehicle's semi-active controller [13].

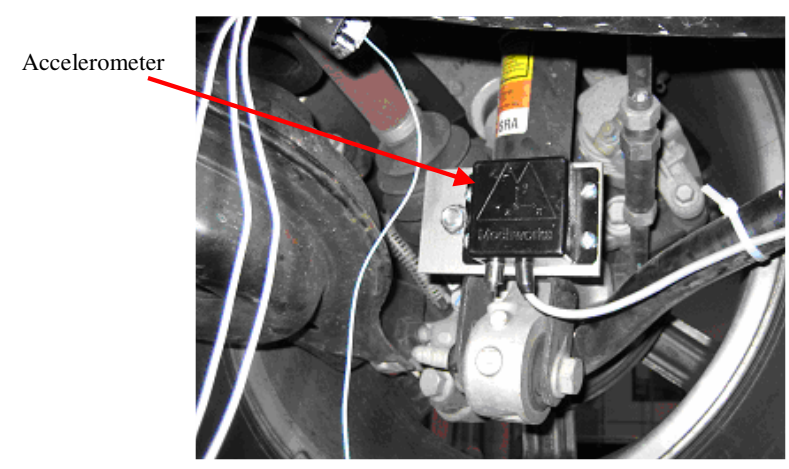

Fig. 6. The accelerometer mounted at the wheel end of the shock. 
The control problem is defined as follows. Being available the MagneRide ${ }^{\mathrm{TM}}$ MR damper with given characteristics, it is desired to find the lower and upper bounds of the Skyhook Control Strategy $c_{\min }$ and $c_{\max }$ such that the next requirements are fulfilled

- A soft ride on even roads/pavements

- A controlled ride on uneven roads/bumps with a settling time of around $1 \mathrm{sec}$ and a damping ratio of approximately $\zeta=0.4$ (not more than 2 or 3 bounces).

The aforementioned control requirements are referred to a specific ride quality determined by the designer. By employing the proposed design methodology and using the pole placement technique, $c_{\max }$ is calculated to be around $5500 \mathrm{~N}$. sec/ $\mathrm{m}$. The lower bound of the semi-active damper is also determined by the damper tests to be approximately $c_{\min }=200 \mathrm{~N} . \mathrm{sec} / \mathrm{m}$.

A real-time processing platform running in $\mathrm{VC}++$ was developed to implement the integrated estimator/controller. A Pentium 4 desktop computer with $3.4 \mathrm{GHz}$ computational power (CPU) and $1 \mathrm{~GB}$ instant memory (RAM) was used as the onboard processing machine. The computed control command was sent out of the computer through an RS485 serial port to a voltage controlled current circuit. The circuit drove a PWM amplifier which accordingly fed the vehicle MR dampers. The real time road tests were carried out in the University of Waterloo's ring road where there was a bump. Fig. 7 depicts the bumpy part of the road.

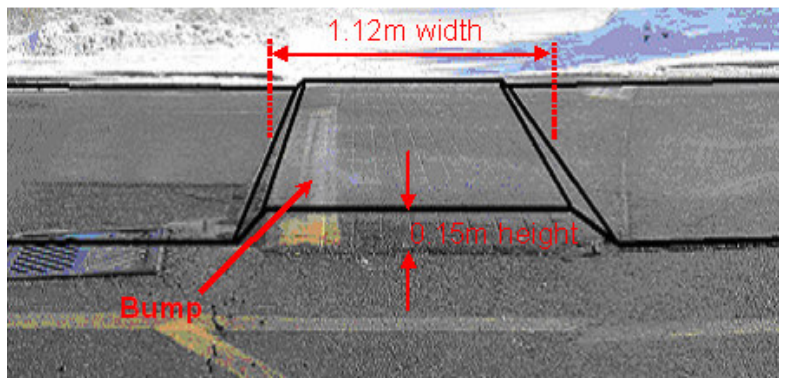

Fig. 7. The bump used during the real time tests.

The test vehicle was driven on the specified section of the ring road with different speeds between $20 \mathrm{~km} / \mathrm{hr}$ to $50 \mathrm{~km} / \mathrm{hr}$. For each velocity, performance of Cadillac SRX original controller was compared with the newly designed semi active controller. Measurements from the vertical axis accelerometer of the IMU mounted near the CG were used as a measure of ride comfort. Fig. 8 shows the acceleration of the $\mathrm{CG}$ for two cases: when using the benchmark control system engaged and when using the designed controller. In comparison with the Cadillac controller, the fuzzy Skyhook controller decreases maximum acceleration and peak to peak acceleration by $19 \%$ and $13 \%$, respectively. However, the settling time increased by $9 \%$. Overall, it was observed that employing the systematic design methodology proposed in this paper results in performance comparable with the benchmark semi-active suspension system. It is clear that with varying the desired settling time and $\zeta$ followed by redesigning the controller, different performance characteristics can be achieved.

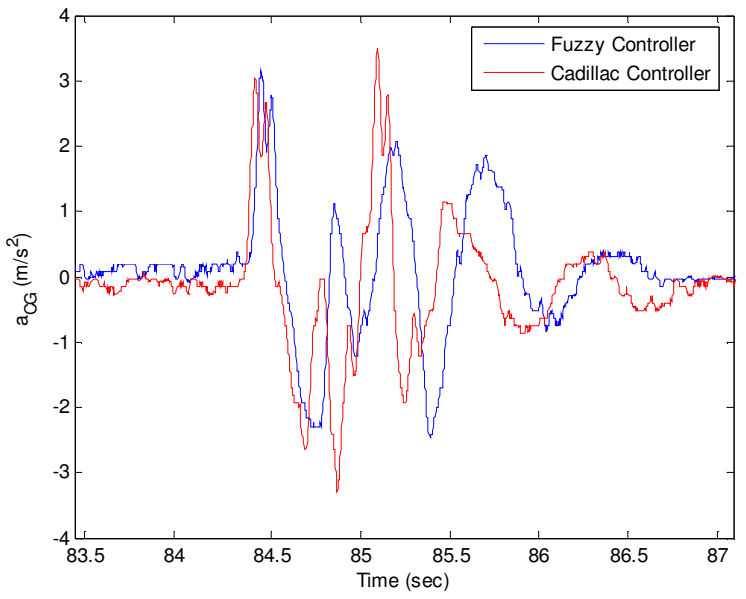

Fig. 8. Accelerations of the vehicle's CG over the bump with $30 \mathrm{~km} / \mathrm{hr}$.

With proper tuning of the membership function parameters in (4) and (5), sensitivity and smoothness of the fuzzy Skyhook controller can be controlled. $\gamma$ is the offset value which is usually set to a small value greater than zero in order to decrease sensitivity of the fuzzy network to input noises. The greater the $\gamma$, the controller becomes less sensitive to the noises as well as small inputs. $q$ changes the membership function growth or decay rate. Setting $q$ to small quantities increases sharpness of the fuzzy controller. The fuzzy Skyhook controller command along with the controller inputs are exhibited in Fig. 9. It is apparent that the designed fuzzy controller is insensitive to noise. In addition, the low damping value assigned by the controller on the even pavement provides a better isolation from the road disturbances and consequently a softer ride.

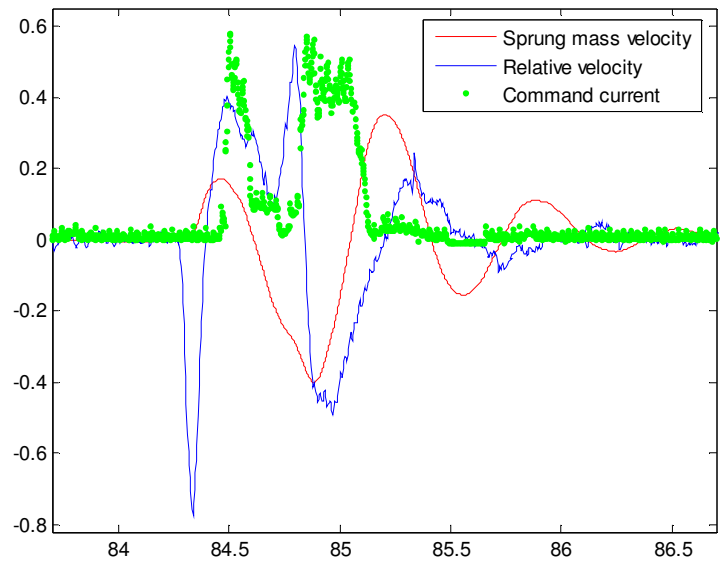

The membership functiome (s arameters can be also

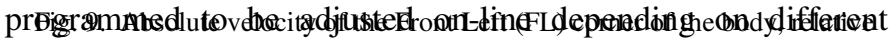
roveldscity of the FL shock and the control current sent to the MR damper. 


\section{CONCLUSION}

A systematic while practical methodology is developed to design vehicles semi-active suspension control systems. The proposed methodology maps the original nonlinear and discontinuous problem into a new space where the wellestablished conventional control techniques can be employed. The controller policy which is offered by the proposed procedure is simple with low computational burden and hence straight forward for implementation on a low cost microcontroller. Real time road tests demonstrate effectiveness of the proposed design methodology.

\section{ACKNOWLEDGMENT}

Authors would like to thank the Phoenix research team. Also, financial support provided by Mechworks Inc. and Materials and Manufacturing Ontario (MMO) is appreciated.

\section{REFERENCES}

[1] D. Karnopp, M. J. Crosby and R. A. Harwood, "Vibration Control Using Semi-Active Force Generators," ASME J. Eng. Ind., No. 2, pp. 619-626, 1974.

[2] M. Novak and M. Valasek, "A New Concept of Semi-Active Control of Trucks Suspension," Proceeding of AVEC 96, International Symposium on Advanced Vehicle Control, Aachen University of Technology, pp. 141-151, 1996.

[3] K. Hong, H. Sohn and J. K. Hedrick, "Modified Skyhook Control of Semi-Active Suspensions: A New Model, Gain Scheduling, and Hardware in the Loop Tuning," ASME J. of Dynamic Systems, Measurement, and Control, Vol. 124, pp. 158-167, March 2002.

[4] Y. Liu, F. Gordaninejad, C. Evrensel, S. Karakas, U. Dogruer, "Experimental study on fuzzy skyhook control of a vehicle suspension system using a magneto-rheological fluid damper," Smart Structure and Material: Industrial and Commercial Applications of Smart Structure Technologies, Proceeding of SPIE, Vol. 5388, pp. 338-347, 2004.

[5] M. Ahmadian, F. D. Goncalves, C. Sandu, "An Experimental Analysis of Suitability of Various Semi-Active Control Methods for Magnetorheological Vehicle Suspensions," Smart Structure and Material: Damping and Isolation, Proceeding of SPIE, Vol. 5760, pp. 208-222, 2005.

[7] M. Ahmadian, "On the Development of Fuzzy Skyhook Control for Semi-active Magneto Rheological Systems," Smart Structure and Material: Damping and Isolation, Proceeding of SPIE, Vol. 5760, pp. 268-282, 2005.

[8] J. Lieh and W. J. Li, "Adaptive Fuzzy Control of Vehicle Semi-Active Suspension Control," ASME J. of Dynamic Systems, Measurement, and Control, Vol. 61, pp. 293-297, 2004.

[9] D. E. Ivers and L. R. Miller, "Experimental Comparison of Passive, Semi-Active On/Off, and Semi-Active Continuous Suspensions," SAE technical paper series No. 892484.

[10] D. Sammier, O. Sename and L. Dugard, "Skyhook and $\mathrm{H}_{\infty}$ Control of Semi-active Suspensions: Some Practical Aspects," Vehicle System Dynamics, Vol. 39, No. 4, pp. 279-308, 2003.

[11] F. D. Goncalves, J. Koo and M. Ahmadian, "Experimental Approach for Finding the Response Time of MR Dampers for Vehicles Applications," Proceeding of DETC'03: ASME 2003 Design Engineering Technical Conferences and Computers and Information in Engineering Conference. Chicago, Illlinois USA, September 2-6, 2003.

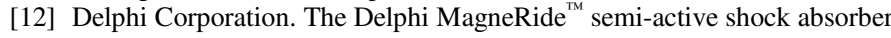
.http://www.delphi.com

[13] H. Bolandhemmat, C. Clark, F. Golnaraghi, "A Distributed Sensing System for Vehicles States Estimation,” Proceedings of IMECE2006, ASME International Mechanical Engineering Congress and Exposition. Chicago, Illinois, USA. November 5-10, 2006
[14] Li-Xin Wang, A Course in Fuzzy Systems and Control, $2^{\text {nd }}$ ed., Prentice Hall, 1997.

[15] L. A. Zadeh, "Outline of a new approach to the analysis of complex systems and decision processes," IEEE Transaction on Systems, Man and Cybernetics, Vol. 3, pp. 28-44, 1973.

[16] SRX Standard and Optional Features, http://gmcanada.com. 